\title{
Looking back, moving forward: 50 years of South African Medical Research Council alcohol-related publications
}

\author{
CD Parry, ${ }^{1,2} \mathrm{PhD}$; B Myers, ${ }^{1,3} \mathrm{PhD}$; R Matzopoulos, ${ }^{4,5} \mathrm{PhD}$; N Morojele, ${ }^{5,7,8} \mathrm{PhD}$; N Siegfried, ${ }^{1,3} \mathrm{MB}$ ChB, DPhil \\ ${ }^{1}$ Alcohol, Tobacco and Other Drug Research Unit, South African Medical Research Council, Cape Town, South Africa \\ ${ }^{2}$ Department of Psychiatry, Faculty of Medicine and Health Sciences, Stellenbosch University and Tygerberg Hospital, South Africa \\ ${ }^{3}$ Department of Psychiatry and Mental Health, Faculty of Health Sciences, University of Cape Town, South Africa \\ ${ }^{4}$ Burden of Disease Research Unit, South African Medical Research Council, Cape Town, South Africa \\ ${ }^{5}$ School of Public Health \& Family Medicine, University of Cape Town, South Africa \\ ${ }^{6}$ Alcohol, Tobacco and Other Drug Research Unit, South African Medical Research Council, Pretoria, South Africa \\ ${ }^{7}$ School of Public Health, Faculty of Health Sciences, University of the Witwatersrand, Johannesburg, South Africa \\ ${ }^{8}$ Department of Psychology, University of Johannesburg, South Africa
}

Corresponding author: C D Parry (cparry@mrc.ac.za)

Background. Alcohol is one of the highest risk factors for death and disability in South Africa (SA).

Objective. To explore the trajectory of empirical research on alcohol in SA between 1969 and 2019, with an emphasis on South African Medical Research Council (SAMRC) authored publications.

Methods. We reviewed published research (Pubmed and Africa-Wide Information) using systematic methods, clear inclusion and exclusion criteria, and defined search terms. The search was not limited by language. Data synthesis was carried out by the first and last authors.

Results. A total of 867 journal articles met the inclusion criteria, with $243(28.0 \%)$ authored or co-authored by SAMRC researchers. For the latter group, three-quarters had an SAMRC researcher as first or last author. Over three-quarters (78.6\%) of the SAMRC author positions ('first', 'last' or 'other, counting researchers from a unit only once, but counting authors across different units on a single publication) were from intramural units. Over half the articles authored by SAMRC researchers focused on non-communicable diseases (55.9\%), 23.8\% focused on communicable diseases, and $10 \%$ on crime, violence or injury. Few articles focused on alcohol and tuberculosis (TB), alcohol and cancer, or alcohol policy. Over three-quarters (76.9\%) were epidemiological in nature, and $65.3 \%$ were cross-sectional studies. There were 17 reviews (7 systematic) and 11 randomised controlled trials (RCTs). There was an increase in the annual number of publications over the 50-year period for both SAMRC and non-SAMRC researchers. Over time, there has been a trend towards publishing on alcohol research in journals published outside SA, but the SAMJ still remains a popular journal choice.

Conclusion. The SAMRC has contributed substantially to the growing field of alcohol research in SA, but gaps in areas such as alcohol policy evaluation, alcohol and its association with TB and cancer, and interventional research, are evident.

S Afr Med J 2019;109(11 Suppl 1):S30-S35. https://doi.org/10.7196/SAMJ.2019.v109i11b.14277

A prime objective of national research institutes such as the UK Medical Research Council (MRC-UK) and the US National Institutes of Health (NIH) is to stimulate the undertaking of public health research and ensure that research findings are disseminated through publications, conference presentations and other media. This is typically achieved by soliciting Requests for Proposals and Requests for Applications, as well as through funding intramural research units and extramural research centres based at universities. The South African Medical Research Council (SAMRC), a statutory research body with a broad mandate similar to the MRC-UK and NIH, has followed this model.

In 2001, the Alcohol and Drug Abuse Research Unit (renamed the Alcohol, Tobacco \& Other Drug Research Unit in 2013) of the SAMRC was established to respond to the growing recognition of the health and societal burden of alcohol and drug use in the country. ${ }^{[1]}$ Before the unit was established, SAMRC research on alcohol issues was undertaken by intramural and extramural research units that were not focused on substance use but which included research in this area. Intramural research units at the SAMRC have been largely set up to address health issues with a major impact on South Africa (SA) in terms of their effect on burden of disease or associated risk factors. Alcohol is the 5th-largest risk factor for death and disability in SA, ${ }^{[2]}$ and in $2015 \sim 62300$ (95\% uncertainty interval (UI) 27000 - 103000 ) adults died in this country from alcohol-attributable causes. ${ }^{[3]}$ Alcohol consumption is roughly $50 \%$ higher in SA than in the WHO African region as a whole, and while many South Africans abstain from drinking alcohol, levels of heavy episodic drinking are very high among male and female drinkers (71\% and $34 \%$, respectively). ${ }^{[4]}$

A wide variety of alcohol-related research studies have been conducted by SAMRC scientists, both intra- and extramural, between 1969 and 2019, but little is known about the trajectory of this research. In particular, with regard to published research, little is known about whose work is being published at the SAMRC (early-, mid- or late-career scientists), how the scope of research has unfolded over time, what kinds of intervention studies have been carried out, whether scientific outputs have been increasing over time, and what changes have taken place in terms of the research funding sources. Such reflection is useful in assessing the value of SAMRC research on this important topic and to give guidance on how it could be strengthened in the future. This review is of published empirical research with alcohol as a focus, conducted in 
SA by researchers affiliated with SAMRC intra- and extramural units between 1969 and 2019.

\section{Methods \\ Design}

We undertook a review of published research using systematic methods after developing a protocol (available from authors but mirrors what is in Methods sub-sections below).

\section{Search strategy}

We developed a search strategy in consultation with the SAMRC librarian. The search comprised free-text terms for 'alcohol*', 'substance abuse', 'substance use', 'drink*', 'drunk', 'drunken', 'intoxicated', and 'alcoholic beverage*' combined with 'South Africa'. Comprehensive searches of PubMed, and Africa Wide Information (via EbscoHost), were undertaken to identify publications. The search was conducted in April 2019 and was not limited by language. The search strategy is available on request.

\section{Inclusion criteria}

We included published journal articles of research studies and scientific letters reporting on research findings with a specific focus on alcohol in the context of SA. Articles which focused on SA alone or SA and other countries were therefore deemed eligible. Articles which had alcohol in the title, in the aims or objectives or as a key word were considered alcohol-focused. Both animal and human studies were eligible for inclusion.

\section{Exclusion criteria}

Non-scientific letters and commentaries were excluded, as well as book reviews, meeting reports, editorials, protocols and obituaries. All publications which did not have a specific focus on alcohol and which did not include any focus on SA were excluded as were nonempirical research articles. Articles which included alcohol as only one among several co-variates or explanatory variables to be included in analyses were not deemed as having a specific focus on alcohol and were excluded. We also excluded case studies with less than 10 cases. Newspapers and magazines were also excluded as were studies looking at the chemical properties of wine.

\section{Study selection and data extraction}

One investigator (CP) independently selected potentially eligible publications from the records yielded in the searches. While the focus was on publications authored by SAMRC intramural and extramural scientists, the initial search encompassed all articles focusing on alcohol and SA in order to get a denominator for publications on alcohol and SA. EndNote was used, with Author Address and the Notes fields being used to identify articles authored by researchers at the (SA)MRC (or (SA)MNR or (South African) Medical Research Council or Suid-Afrikaanse Mediese Narvorsingsraad.

\section{Data synthesis}

CP selected articles according to the inclusion and exclusion criteria above and created an MS Excel spreadsheet documenting which articles were authored/co-authored by SAMRC staff, whether they were intra- or extramural staff or both, the SAMRC units to which the SAMRC authors were affiliated and whether the SAMRC authors had first author and/ or senior author position or were one of the middle authors. CP and NS used a coding sheet to extract data from the detailed EndNote fields and abstracts of each included article. The content focus of each study was classified as: (i) communicable disease (HIV, TB, other), (ii) non-communicable disease (NCDs: cancer, fetal alcohol spectrum disorder, liver, mental health, other), (iii) violence and injury, and (iv) other. Research focus was categorised as (i) epidemiology, (ii) treatment, or (iii) prevention, and the study design as qualitative, case study, cross-sectional, cohort, case-control, RCT, and systematic review. Additional categories were added as needed and any disagreements were addressed through discussion until agreement was reached. Data were analysed using descriptive statistics and graphical displays of the data.

\section{Results}

Number of articles by intraand extramural SAMRC units and analysis of the number of publications over time

Over 5600 journal articles on the topic of alcohol from empirical studies and including a focus on SA were identified in the initial scoping, 3957 from PubMed and 1647 from Africa-Wide Information (Fig. 1). After removing duplicates, articles with no author, articles where there was no mention of alcohol in the abstract, and articles excluded for other reasons, 867 journal articles met inclusion criteria, with 243 (28.0\%) authored or co-authored by SAMRC intramural and extramural researchers (http://www.samj. org.za/public/sup/14277.pdf). Between 1969 and 2019, the number of publications on alcohol and SA increased substantially over time (Fig. 2). For the early years of the SAMRC's existence (1969 - 1993), few articles on alcohol were authored by SAMRC researchers, but after 1999, articles authored by SAMRC researchers increased substantially.

Table 1 gives the number of authored publications by SAMRC unit name, whether the unit was intra- or extramural and author position (first author, senior author if not first author, and other author position if not

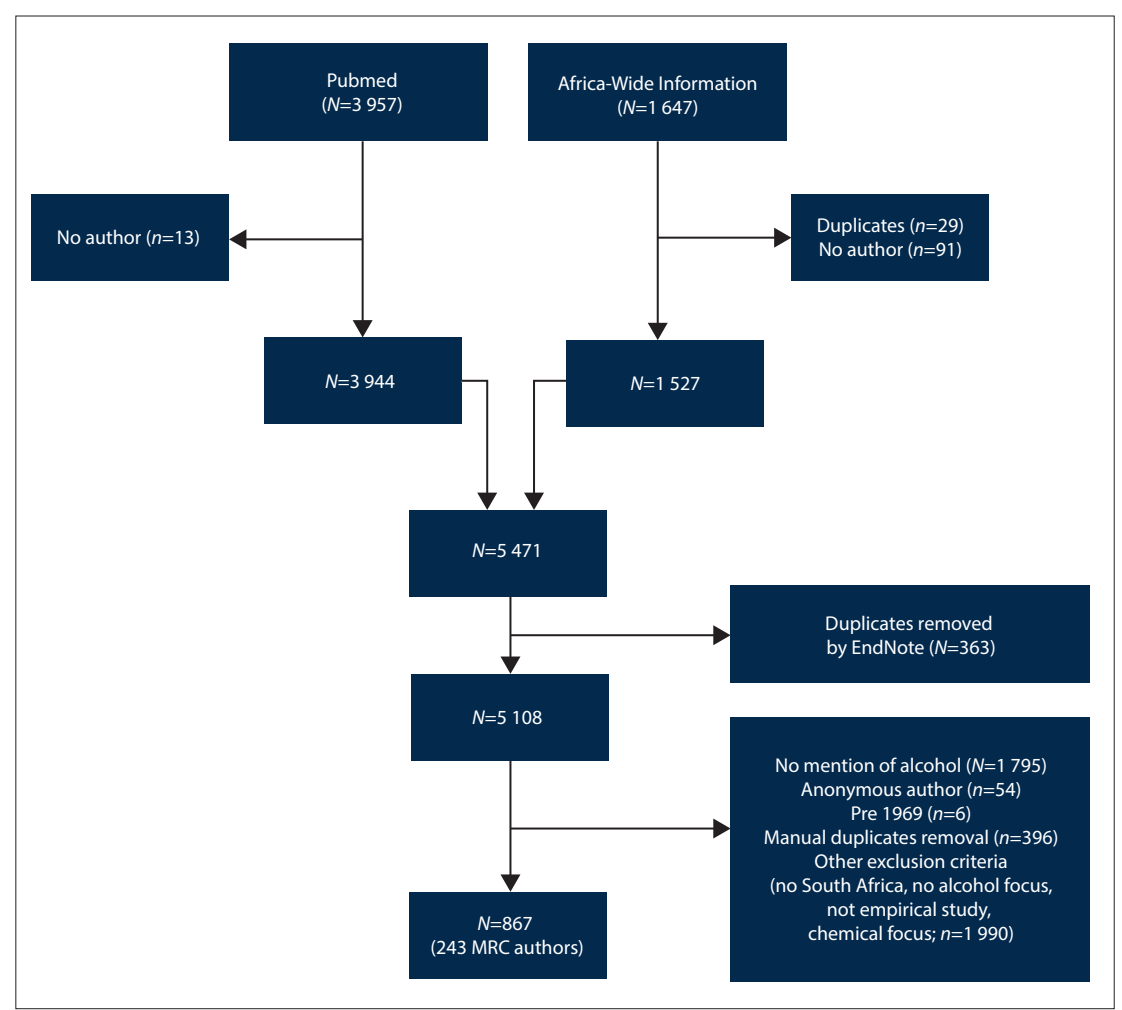

Fig. 1. PRISMA diagram of search process and outcome. 
Table 1. Authored publications by unit name, type, and author position ${ }^{\star}$

\begin{tabular}{|c|c|c|c|c|}
\hline Unit & $\begin{array}{l}\text { First author } \\
\text { publications, } n^{\dagger}\end{array}$ & $\begin{array}{l}\text { Senior author publications } \\
\text { if not first author, } n^{\dagger}\end{array}$ & $\begin{array}{l}\text { Publications where not first } \\
\text { or senior author, } n^{* \dagger}\end{array}$ & Total, $n$ \\
\hline \multicolumn{5}{|l|}{ Intramural units } \\
\hline ATODRU (formerly ADARU) & 84 & 15 & 44 & 143 \\
\hline BRU (formerly Institute for Biostatistics) & 1 & 5 & 28 & 34 \\
\hline BODRU & 2 & 0 & 3 & 5 \\
\hline CDLRU & 3 & 0 & 1 & 4 \\
\hline CERSA (and CHRG) & 2 & 2 & 0 & 4 \\
\hline Cochrane Centre & 0 & 0 & 2 & 2 \\
\hline GHRU & 3 & 0 & 2 & 5 \\
\hline HPRU & 1 & 0 & 1 & 2 \\
\hline HSRU & 6 & 1 & 4 & 11 \\
\hline National TB Research Programme & 1 & 0 & 0 & 1 \\
\hline NCDRU & 0 & 1 & 0 & 1 \\
\hline MRU & 1 & 0 & 0 & 1 \\
\hline RIND & 2 & 0 & 0 & 2 \\
\hline TRU & 0 & 0 & 1 & 1 \\
\hline VIPRU (formerly CVIRU, NTRP) & 7 & 1 & 6 & 14 \\
\hline NUHRP & 5 & 0 & 1 & 6 \\
\hline \multicolumn{5}{|l|}{ Extramural units } \\
\hline BMRG (Wits) & 1 & 0 & 0 & 1 \\
\hline UCAH (UCT) & 0 & 3 & 0 & 3 \\
\hline DPHRU (Wits) & 1 & 0 & 0 & 1 \\
\hline HGRU (UCT) & 3 & 0 & 0 & 3 \\
\hline MIRU (UCT) & 17 & 1 & 2 & 20 \\
\hline RUESSM (UCT) & 1 & 0 & 0 & 1 \\
\hline MMRU (Wits) & 0 & 0 & 1 & 1 \\
\hline MHRU (Wits) & 1 & 0 & 0 & 1 \\
\hline RPHHTRU (Wits) & 0 & 2 & 1 & 3 \\
\hline HCDRU (NWU) & 2 & 0 & 2 & 4 \\
\hline UNMD & 1 & 0 & 0 & 1 \\
\hline URRMD (UCT) (formerly ASDU) & 4 & 3 & 17 & 24 \\
\hline \multicolumn{5}{|c|}{$\begin{array}{l}\text { ATODRU = Alcohol, Tobacco and Other Drug Research Unit, ADARU = Alcohol, Drug Abuse Research Unit; BRU = Biostatistics Research Unit, BODRU = Burden of Disease Research Unit; } \\
\text { CDLRU = Chronic Diseases of Lifestyle Research Unit; CERSA = Centre for Epidemiological Research in Southern Africa; CHRG = Community Health Research Group; GHRU = Gender and } \\
\text { Health Research Unit; HPRU = Health Promotion Research Unit; HSRU = Health Systems Research Unit; NCDRU = Non-Communicable Diseases Research Unit; MRU = Malaria Research } \\
\text { Unit; RIND = Research Institute for Nutritional Diseases; TRU = Tuberculosis Research Unit; VIPRU = Violence, Injury and Peace Research Unit; CVIRU = Crime, Violence \& Injury Research } \\
\text { Unit; NTRP = National Trauma Research Programme; NUHRP = National Urbanisation and Health Research Programme; BMRG = Brain Metabolism Research Group; Wits = University of } \\
\text { the Witwatersrand; UCAH = Unit on Child and Adolescent Health; UCT = University of Cape Town; DPHRU = Developmental Pathways for Health Research Unit; HGRU = Human Genetics } \\
\text { Research Unit; MIRU = Medical Imaging Research Unit; RUESSM = Research Unit for Exercise Science and Sports Medicine; MMRU = Mineral Metabolism Research Unit; MHRU = Molecular } \\
\text { Hepatology Research Unit; RPHHTRU = Rural Public Health and Health Transitions Research Unit; HCDRU = Hypertension and Cardiovascular Disease Research Unit; NWU = North-West } \\
\text { Univeristy; UNMD = Unit for the Neurochemistry of Mental Diseases; URRMD = Unit on Risk and Resilience in Mental Disorders; ASDU = Stress Anxiety and Stress Disorders Unit. } \\
\text { ×If there is more than one author per unit in the other' category, the article was only counted once. The number of publications in Table } 1 \text { is }>243 \text {, as some articles were authored by persons from } \\
\text { more than one unit. } \\
\text { 'Unless otherwise specified. }\end{array}$} \\
\hline
\end{tabular}

first or senior author, and not counting multiple positions between first and senior author for authors in the same unit). The majority (61.7\%) of the articles authored by SAMRC researchers had an SAMRC researcher as the first author. A further $14.0 \%$ of the articles where SAMRC researchers were not first authors had SAMRC researchers in senior (last) author position. Of the SAMRC authored articles, a fifth (19.8\%) included researchers across more than one unit, mostly within the intramural space. Eight (3.3\%) articles were authored by researchers from both intra- and extramural units, all involving the Alcohol, Tobacco and Other Drug Research Unit (ATODRU), in partnership with the Anxiety and Stress Disorders Unit (ASDU) ( $n=7)$, or with the ASDU and the Child and Adolescent Health Unit $(n=1)$.

\section{Comparison of publications across intra- and extramural research units}

The majority (78.6\%) of author positions were held by researchers in intramural units, with the remainder $(21.4 \%)$ authored by researchers from extramural units. The intramural units having the highest number of author positions (publications) were ATODRU (47.8\%), followed by the Biostatistics Unit (BIOSTATS; 11.4\%), the Violence, Injury and Peace Research Unit (VIPRU; 4.7\%) and the Health Systems Research Unit (HSRU, 3.7\%) (Table 2). For the extramural units, the highest number of author positions came from the University of Cape Town (UCT) Unit on Risk and Resilience in Mental Disorders (URRMD, 8.0\%) and the UCT Medical Imaging Research Unit (MIRU, 6.7\%) (Table 2).

\section{Analysis of content focus, design and type}

Over half of the articles with SAMRC authors focused on NCDs (55.9\%), with a third of the NCD-related articles comprising research on FASD risk for alcohol-exposed pregnancies (32.9\%), a quarter on substance use disorders (24.5\%), and a fifth on alcohol use disorders (20.3\%) (Table 3, Fig. 3). Communicable diseases comprised a quarter of the articles (23.8\%) and roughly $10 \%$ each 


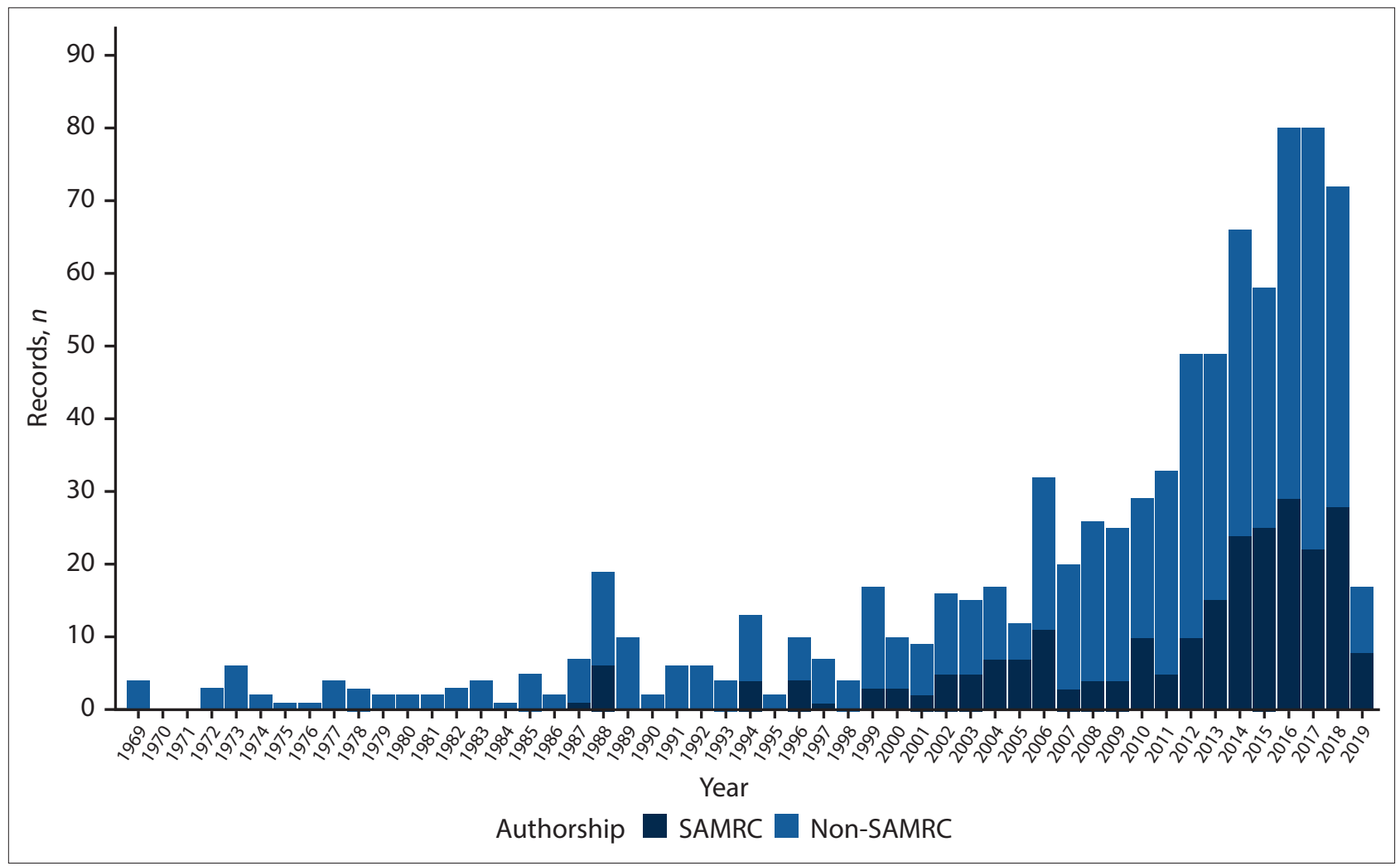

Fig. 2. Number of publications by year: SAMRC-authored v. non-SAMRC authored.

Table 2. Number and proportion of author positions by intra- and extra-mural units having more than $\mathbf{1 0}$ author positions

\begin{tabular}{|c|c|c|}
\hline Unit & Unit type & $\begin{array}{l}\text { Different } \\
\text { author } \\
\text { positions, } \\
n(\%)\end{array}$ \\
\hline ATODRU (formerly ADARU) & Intramural & $143(47.8)$ \\
\hline BRU (Institute for Biostatistics) & Intramural & $34(11.4)$ \\
\hline URRMD (formerly ASDU) UCT & Extramural & $24(8.0)$ \\
\hline MIRU, UCT & Extramural & $20(6.7)$ \\
\hline VIPRU (formerly CVIRU, NTRP) & Intramural & $14(4.7)$ \\
\hline HSRU & Intramural & $11(3.7)$ \\
\hline Other units combined & Intramural & $34(11.4)$ \\
\hline Other units combined & Extramural & $19(6.4)$ \\
\hline \multicolumn{3}{|c|}{$\begin{array}{l}\text { ATODRU = Alcohol, Tobacco and Other Drug Research Unit; ADARU = Alcohol, Drug } \\
\text { Abuse Research Unit; BRU = Biostatistics Research Unit; URRMD = Unit on Risk and } \\
\text { Resilience in Mental Disorders; ASDU = Anxiety and Stress Disorders Unit; } \\
\text { UCT = University of Cape Town; MIRU = Medical Imaging Research Unit, } \\
\text { VIPRU = Violence, Injury and Peace Research Unit; CVIRU = Crime, Violence and Injury } \\
\text { Research Unit; NTRP = National Trauma Research Programme; HSRU = Health Systems } \\
\text { Research Unit. }\end{array}$} \\
\hline
\end{tabular}

on crime/violence/injury and 'other topics'. Of the articles in the communicable diseases category, 4 had a focus on alcohol and TB. In the NCD category there were 2 articles on alcohol and cancer, and in the 'other' category there were 6 having an alcohol policy focus.

Over three-quarters (76.9\%) of the SAMRC-authored articles were epidemiological in research focus, with $9.1 \%$ being treatment-oriented studies and $7.4 \%$ being prevention-oriented (Table 4). Less than $1 \%$ comprised basic science. In terms of study design, the most common type was cross-sectional (57\%), with a further $8.3 \%$ being classified as controlled cross-sectional studies (Table 5). Almost $9 \%$ of the studies were qualitative in nature. Seven percent of the studies $(n=17)$ were review studies, with just under half of them being systematic

\begin{tabular}{ll} 
Table 3. Breakdown of articles by content focus & \\
\hline Area & $\boldsymbol{n}(\%)$ \\
\hline Communicable diseases & \\
HIV & $51(19.9)$ \\
Other communicable ${ }^{*}$ & $10(3.9)$ \\
NCD & \\
$\quad$ FASD/or risk for alcohol-exposed pregnancies & $47(18.4)$ \\
$\quad$ Mental health & $17(6.6)$ \\
$\quad$ Alcohol use disorders & $29(11.3)$ \\
$\quad$ Substance use disorders & $35(13.7)$ \\
$\quad$ Other NCD & $15(5.9)$ \\
Crime, violence or injury & $29(11.3)$ \\
Other topics & \\
FASD $=$ fetal alcohol spectrum disorders, NCD = non-communicable diseases.
\end{tabular}

review studies $(n=7 / 17)$. $^{[11, \$ 15, \$ 37, \$ 59, \$ 180, \$ 204, \$ 213]}$ There were 2 Cochrane Systematic Reviews, ${ }^{[1515,5204]}$ one on school-based interventions and one on banning or restricting alcohol advertising. Eleven articles (4.5\%) described randomised controlled trials (RCTs), covering a wide variety of topics such as alcohol interventions in the workplace $(n=1)$, alcohol interventions in emergency care settings $(n=3)$, alcohol-related HIV interventions $(n=3)$, a pharmacotherapy intervention to address alcohol-dependence $(n=1)$, an intervention to address alcohol-related hereditary haemochromatosis and dietary iron overload, and alcoholrelated interventions to reduce the impact of drinking on fetuses $(n=2)$.

\section{Journals in which articles were published, and the agencies funding the research}

SAMRC authors published the 243 articles in 127 journals between 


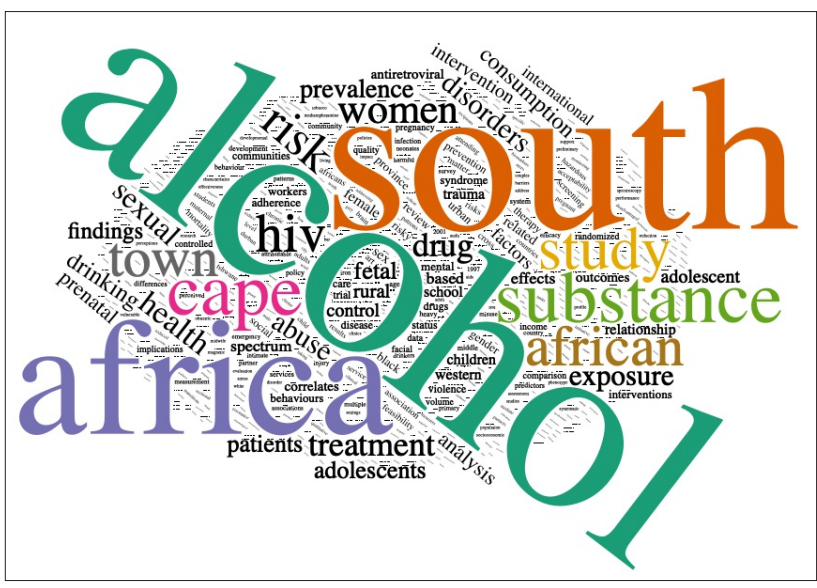

Fig. 3. Word cloud by title of articles co-authored by SAMRC researchers (1969-2019).

Table 4. Breakdown of articles by research focus (SAMRCauthored publications) $(N=222)$

\begin{tabular}{ll} 
authored publications) $(\mathbf{N = 2 2 2})$ & $\boldsymbol{n}(\mathbf{\%})$ \\
\hline Epidemiology & $185(76.9)$ \\
Diagnostic & $17(7.0)$ \\
Prevention & $17(7.0)$ \\
Basic science & $2(0.8)$ \\
Policy review & $1(0.4)$
\end{tabular}

Table 5. Breakdown of articles by study design

\begin{tabular}{ll}
\hline Study type & $\boldsymbol{n}(\%)$ \\
\hline Cross-sectional & $140(57.6)$ \\
Qualitative & $21(8.6)$ \\
Controlled cross-sectional & $21(8.6)$ \\
Cohort & $12(4.9)$ \\
Case-control & $11(4.5)$ \\
RCT & $11(4.5)$ \\
Review & $10(4.1)$ \\
Systematic review & $7(2.9)$ \\
Non-controlled before after & $3(1.2)$ \\
Controlled before after & $2(0.8)$ \\
Feasibility for clinical trial & $2(0.8)$ \\
Other * & $3(1.2)$ \\
RCT $=$ randomised controlled trial. &
\end{tabular}

1969 and 2019. Table 6 contains a list of the journals in which empirical articles have been published 4 or more times over the 50 years. The most popular journals in which articles were published were the South African Medical Journal (SAMJ; $n=14 ; 5.8 \%$ ), Substance Abuse Treatment Prevention and Policy ( $n=11 ; 4.5 \%), A I D S$ and Behavior, Alcoholism Clinical and Experimental Research (ACER) and Journal of Studies on Alcohol and Drugs ( $n=9 ; 3.7 \%$ each). In terms of trends over time, initially there was a focus on publishing in local journals like the SAMJ and non-indexed journals such as the Urbanisation and Health Newsletter, the MRC Trauma Review and the African Journal of Drug and Alcohol Studies. SAMRC intra- and extramural researchers have continued to publish in the SAMJ, but there has been an increase in recent years in publishing in international, ISIindexed journals such as ACER, Drug and Alcohol Dependence, Drug and Alcohol Review, PLoS ONE, Patient Preference and Adherence, Substance Abuse Treatment Prevention and Policy (STPP) and in the BMC journals $(n=15)$. Only a few publications $(n=10)$ were published
Table 6. List of journals having 4 or more publications by SAMRC staff (1969 - 2019)

\begin{tabular}{ll}
\hline Journal & $n$ \\
\hline South African Medical Journal & 14 \\
Substance Abuse Treatment Prevention and Policy & 11 \\
AIDS and Behaviour & 9 \\
Alcoholism Clinical and Experimental Research & 9 \\
Journal of Alcohol and Drug Studies & 9 \\
African Journal of Drug and Alcohol Studies & 8 \\
AIDS Care & 8 \\
Drug and Alcohol Review & 7 \\
BMC Psychiatry & 5 \\
Drug and Alcohol Dependence & 5 \\
Addiction & 4 \\
BMJ Open & 4 \\
Curationis & 4 \\
Human Behavior Mapping & 4 \\
Metabolic Brain Disease & 4 \\
PLoS ONE & 4 \\
Urbanisation and Health Newsletter (Urban Heath & 4 \\
and Development Bulletin) & \\
SAMrC = South African Medical Research Council. & \\
&
\end{tabular}

in higher impact factor journals, such as Addiction, BMC Medicine, Cochrane Database of Systematic Reviews and the Lancet. Information on funding of the research was not able to be obtained from the metadata extracted by Endnote.

\section{Discussion}

The SA alcohol research studies conducted over the 50-year period of the SAMRC's existence reported in this review, reflect a relatively strong area of research, and one which is growing in terms of outputs, particularly over the last $10-15$ years. This is appropriate in terms of the high burden of harm associated with alcohol use in SA, and the growing recognition of the need for national and global action to reduce harm related to alcohol use. ${ }^{[1-4]}$ With almost $30 \%$ of the publications being authored or co-authored by SAMRC intramural and extramural researchers, and with $76 \%$ in first or last author position, SAMRC intra- and extramural researchers are making a substantial contribution to knowledge regarding alcohol in SA. It should be noted that the SAMRC also provides funds for short-term research on alcohol where there have been requests for proposals, and as a result the SAMRC is likely to have funded some of the research authored by non-SAMRC researchers included in the 867 publications. It would be useful in future to consider the amount of funding that has gone into supporting the research over time to determine the cost-per-publication and whether this has changed over time.

There appears to be substantial collaboration across intramural units and across extramural units in the SAMRC, with a fifth of the publications including researchers across more than one unit. However, with only 7 articles authored by researchers across both intra- and extramural units, there is scope for more collaboration across such research entities. Funding mechanisms could potentially be instituted by the SAMRC to incentivise this.

The 2010 Science, Engineering and Technology Institution (SETI) Review $^{[5]}$ of the SAMRC proposed that funds might be better spent by the SAMRC supporting extramural units over intramural units. Counter to this, our findings show that intramural units have made a significant contribution in terms of publishing the findings of empirical research in the alcohol field. However, the greater amount of 
publications by intramural researchers in this area could also be partly due to extramural units not having an obligation or economic incentive to list their SAMRC affiliation on publications until around 2013. It is furthermore not surprising that the ATODRU had the highest number of publications/author positions, given the substantial focus on alcohol by this unit, established in 2001. ${ }^{[1]}$ The relatively high number of publications involving researchers in BIOSTATS and VIPRU is also understandable, given the important support role played by the former and the high link between alcohol and crime, violence, and injury. ${ }^{[6]}$ The high number of papers from the top two extramural units is also not surprising, given the high comorbidity between mental disorders and alcohol, and the growing field of neuroscience.

The breakdown of articles by study focus, with over half the articles on NCDs and a quarter on infectious disease, reflects the burden of death and disability in SA in terms of both causes of death and disability and also risk factors for these causes, ${ }^{[2,7]}$ It also reflects the interest of local and international researchers and the availability of funding from the US Centers for Disease Control and Prevention, the US National Institutes of Health, the SAMRC, the National Research Foundation and other funders. Despite the possibly closer causal linkage between alcohol and TB as compared ith alcohol and HIV, ${ }^{[6]}$ very few published studies were found on alcohol and TB, suggesting that this may be an area where more research is needed in South Africa, both to clarify the ways in which alcohol use affects the incidence of $\mathrm{TB}$ in this country as well as treatment outcomes and how effects can be mitigated. The proportion of papers on the topic of alcohol and injury is probably low, considering the burden of injury in SA and the link to alcohol, and this should probably be given greater emphasis in future research. ${ }^{[7,8]}$ In addition, few articles focused on alcohol and cancer or on alcohol control policies at a macro level. The connection between alcohol and cancer has recently been highlighted in international research ${ }^{[9]}$ and an umbrella review of systematic reviews of alcohol control policies conducted by the SAMRC found no primary research studies evaluating the effect of macro-level alcohol policy interventions from LMICs. ${ }^{[10]}$

The finding that most of the studies were epidemiological in nature and that less than $16 \%$ of the studies had a treatment or prevention focus also raises questions around whether there needs to be more emphasis on intervention research, especially given that much is now known about the burden of death and disability associated with alcohol in SA and populations at risk. The lack of intervention research is clearly demonstrated by the fact that only 11 RCTs reported on in papers were authored by SAMRC researchers. There has, however, been a shift in research focus, with $25 \%$ of the studies categorised as being treatment- or prevention-oriented since 2016.

The fact that SA researchers are publishing in a range of international journals is encouraging, as is the fact that there has been a move away from publishing in non-indexed journals. The most popular journals in which articles were published were the SAMJ and SATPP. The former is the leading medical/public health journal in SA and SATPP is a newer, online journal that has also been receptive to publishing articles on SA.

This review has significant strengths including following systematic methods with preparation of an a priori protocol, use of a comprehensive search strategy which was not limited by language, and independent coding conducted by two investigators. Notwithstanding, there are limitations and it should be noted that we did not access full copies of each article to identify further information of relevance such as source of funding. In the current environment of transformation, identification of author qualifications, experience and career progression may have been informative to monitor trends towards racial and gender redress, although this information is often lacking from article citations. Furthermore, the initial selection was only undertaken by one person (CP) and this could have introduced selection bias, but given his close involvement as a staff member of the SAMRC between 1982 and 1984 and since 1990, this can also be viewed as lending additional reliability to classification of author affiliations. Limiting the search to PubMed and AfricaWide Information was pragmatic, but it is possible that additional publications might have been found had we included additional relevant databases such as PsychInfo, for example.

\section{Conclusion}

The study has highlighted the fact that over the past 50 years a great deal of empirical research on the topic of alcohol use in SAhas been conducted and published by SAMRC researchers, particularly within the intramural environment. While the focus of the research has been broad in terms of infectious diseases and NCDs, clear gaps in areas such as the evaluation of alcohol policy and the relationships between alcohol and TB and alcohol and cancer are evident and need addressing in future. In terms of methodology, the research has been largely epidemiological and cross-sectional in nature. Given the health needs of the country, there now needs to be a shift towards building a robust local evidence base through the conduct of rigorous interventional studies to best prevent and treat alcohol-related conditions, and to ensure the creation of an evidence-informed enabling policy environment.

\section{Acknowledgements. None.}

Author contributions. CP and NS designed the study with comments from NM and BM. CP did the initial scoping of articles and he and NS independently rated the SAMRC authored articles on the three areas of content focus, research focus and study design and reached consensus where there was disagreement. RM, NM, BM and NS gave input on ways to analyse the data. CP prepared the tables and figures and prepared the first draft of the manuscript and the other authors made revisions to the manuscript.

Funding. We acknowledge funding from the South African Medical Research Council for covering the salaries of the authors and thereby facilitating their preparation of this manuscript.

Conflicts of interest. None.

1. Parry C, Morojele N, Myers M, Plüddemann A. The Alcohol \& Drug Abuse Research Unit at the Parry C, Morojele N, Myers M, Plüdemann A. The Alcohol \& Drug Abuse Research Unit at the South African Medical Research Council - strengthening substance abuse research and

2. GBD 2015 Risk Factors Collaborators. Global, regional and national comparative risk assessment of 79 2. GBD 2015 Risk Factors Collaborators. Global, regional and national comparative risk assessment of 79
behavioural, environmental and occupational, and metabolic risks or clusters of risks in 195 countries, 1990-2015: A systematic analysis for the Global Burden of Disease Study 2015. Lancet 2016;388:16591724. https://doi.org/10.1016/S0140-6736(16)31679-8

3. Probst C, Parry $\mathrm{CDH}$, Wittchen H-U, Rehm J. The socioeconomic profile of alcohol attributable mortality in South Africa. BMC Med 2018;16:97. https://doi.org/10.1186/s12916-018-1080-0

World Health Organization. Global Status Report on Alcohol and Health 2018. Geneva: WHO, 2018. 5ETI Review Panel. Final report of the panel for the 2010 SETI Review of the South African Medical Research Council. Cape Town: South African Medical Research Council, 2010.

Rehm J, Gmel GE, Gmel G, et al. The relationship between different dimensions of alcohol use and the burden of disease - an update. Addiction 2017;112(6):968-1001. https://doi.org/10.1111/add.13757 GBD Mortality and Causes of Death Collaborators. Global, regional, and notion GBD Mortality and Causes of Death Collaborators. Global, regional, and national life expectancy, all-cause and cause-specific mortality for 249 causes of death, 1980 - 2015: A systematic analysis for the Global Burden of Disease Study 2015. Lancet 2016;388:1459-1544. https://doi.org/10.1016/S0140-

Schneider M, Norman R, Parry CDH, Plüddemann A, Bradshaw D. Estimating the burden of disease attributable to alcohol in South Africa in 2000. S Afr Med J 2007;97(8 Pt 2):664-672.

9. LoConte NK, Brewster AM, Kaur JS, Merril JK, Arberg AJ. Alcohol and cancer: A statement of the American Society of Clinical Oncology. J Clin Oncol 2018;36(1):83-93. https://doi.org/10.1200/ CO.2017.76.1155

10. Siegfried N, Parry CDH. Do alcohol control policies work? An umbrella review and quality assessment of systematic reviews of alcohol control interventions (2006 - 2017). PLoS ONE 2019;14(4):e0214865. https://doi.org.org/10.1371/journal.pone.0214865 